\title{
On the robustness of $\mathrm{H}$-deficient post-AGB tracks
}

\author{
M. M. Miller Bertolami ${ }^{1,2, \star}$ and L. G. Althaus ${ }^{1,2, \star \star}$ \\ ${ }^{1}$ Facultad de Ciencias Astronómicas y Geofísicas, Universidad Nacional de La Plata, Paseo del Bosque s/n, (1900) La Plata, \\ Argentina \\ 2 Instituto de Astrofísica La Plata, IALP, CONICET \\ e-mail: mmiller, al thaus@fcaglp.unlp.edu.ar
}

Received 7 February 2007 / Accepted 6 May 2007

\begin{abstract}
Aims. We analyze the robustness of H-deficient post-AGB tracks regarding previous evolution of their progenitor stars and the constitutive physics of the remnants. Our motivation is a recent suggestion of Werner \& Herwig (2006, PASP, 118, 183) that previous evolution should be important in shaping the final post-AGB track and the persisting discrepancy between asteroseismological and spectroscopical mass determinations. This work is thus complementary to our previous work (Miller Bertolami \& Althaus 2006, A\&A, 454, 845) and intends to shed some light on the uncertainty behind the evolutionary tracks presented there.

Methods. We compute full evolutionary models for PG 1159 stars taking into account different extramixing (overshooting) efficiencies and lifetimes on the TP-AGB during the progenitor evolution. We also assess the effect of possible differences in the opacities and equation of state by artificially changing them before the PG 1159 stage. Also comparisons are made with the few $\mathrm{H}$-deficient post-AGB tracks available in the literature.

Results. Contrary to our expectations, we found that previous evolution is not a main factor in shaping H-deficient post-AGB tracks. Interestingly enough, we find that only an increase of $\sim 50 \%$ in the intershell opacities at high effective temperatures may affect the tracks as to reconcile spectroscopic and asteroseismologic mass determinations. This forces us to conclude that our previous tracks (Miller Bertolami \& Althaus 2006) are robust enough as to be used for spectroscopic mass determinations, unless opacities in the intershell region are substantially different. Our results, then, call for an analysis of possible systematics in the usually adopted asteroseismological mass determination methods.
\end{abstract}

Key words. stars: AGB and post-AGB - while dwarfs

\section{Introduction}

Post Asymptotic Giant Branch (AGB) stars constitute a shortlived transition stage between AGB stars and white dwarf stars. Among them a minority show H-deficient compositions and are suppossed to be the main progenitors of $\mathrm{H}$-deficient white dwarfs, which account for about $15 \%$ of the white dwarf population (Eisenstein et al. 2006). The group of H-deficient postAGB stars displays a wide variety of surface chemical compositions ranging from almost pure helium envelopes to the helium $(\mathrm{He})$, carbon - $(\mathrm{C})$ and oxygen - $(\mathrm{O})$ rich surface composition of the Wolf Rayet central stars of planetary nebulae ([WC]) and the PG 1159 type stars; see Werner \& Herwig (2006), from now on WH06. The surface composition of the last group resembles the intershell region chemistry of AGB star models when some overshooting at the base of the pulse driven convective zone (PDCZ) is allowed during the thermal pulses (Herwig et al. 1997). For this reason, and also due to the fact that the occurrence of late (i.e. post-AGB) thermal pulses is statistically unavoidable in single stellar evolution modeling (Iben et al. 1983), a late helium shell flash is the most accepted mechanism for the formation of these stars (see, however, De Marco 2002). In this scenario, the remaining thin H-rich envelope is either burnt in a very late helium flash (VLTP) that occurs on the hot white dwarf cooling branch after $\mathrm{H}$ burning has almost ceased, or diluted in a late

\footnotetext{
^ Fellow of CONICET, Argentina.

$\star \star$ Member of the Carrera del Investigador Científico y Tecnológico, CONICET, Argentina.
}

helium flash (LTP) that develops when the H burning shell is still active during the horizontal evolution of the stars in the HR diagram (Herwig 2001).

Roughly a third of PG 1159-type stars exhibit multiperiodic luminosity variations caused by non-radial g-mode pulsations. This has allowed researchers to derive structural parameters in particular the mass of these stars - of individual pulsators by means of asteroseismological studies i.e. by comparing adiabatic pulsation periods with the observed ones - e.g. Kawaler \& Bradley (1994) and more recently Córsico \& Althaus (2006). It is important to mention that for applications requiring accurate values of adiabatic pulsation periods full evolutionary models with a realistic thermal structure should be used. Stellar masses of PG 1159 stars can also be derived by comparing the values of $\log g$ and $\log T_{\text {eff }}$ coming from the fitting of line-blanketed non-LTE model atmospheres to the measured spectra (Werner et al. 1991) with tracks coming from stellar evolution modeling. These two different approaches enable us to compare the derived stellar masses. Although previous spectroscopical mass determinations, based on old H-rich post-AGB models, show relatively good agreement with asteroseismological masses (to about 5\%, WH06, roughly $0.03 M_{\odot}$ ), the development of a new generation of stellar evolution sequences that account for the C- and O- rich surface abundances expected in PG 1159 stars (Herwig et al. 1999) has changed the situation. As mentioned by WH06 the new post-AGB tracks are systematically hotter than the old ones, which leads to lower spectroscopical masses. The new mean spectroscopical mass becomes $0.573 M_{\odot}$, this is 
$0.044 M_{\odot}$ lower than previous values; see Miller Bertolami \& Althaus (2006), from now on MA06. This is at variance with asteroseismological predictions. In fact from Table 3 of WH06 and Table 2 of MA06 the asteroseismological masses are usually $10 \%$ higher than their spectroscopical counterparts, except for the hottest known pulsating PG 1159 star RX J2117.1+3412, the spectroscopical mass of which is more than $20 \%$ higher than the asteroseismological one; see Córsico et al. (2007) for a recent and detailed study of this object. The difference in derived masses is a clear indication of the uncertainties weighting upon the mass determination methods.

In this context, WH06 have recently compared new and old tracks and claimed that the previous evolution on the thermally pulsing AGB (TP-AGB) - particularly the third dredge-up (3DUP) efficiency and mass-loss rates - plays a decisive role in the location of the tracks in the HR and $\log T_{\text {eff }}-\log g$ diagrams during the post-AGB evolution. Specifically, as shown by Herwig et al. (1998), a strong 3DUP changes the evolution of the core mass without altering the evolution of its radius. Consequently the mass-radius relation of the remnants will depend on the previous TP-AGB evolution and, if we accept in the "prediction" of shell homology relations $\left(L_{\text {shell }} \sim M_{\text {core }}^{2} R_{\text {core }}{ }^{-1}\right.$, for $M_{\text {core }} \lesssim 0.8 M_{\odot}$, Herwig et al. 1998), the post-AGB tracks would be accordingly altered. WH06 also point out that mass loss can produce a similar effect as remnants of similar mass may come out with very different degrees of degeneracy depending on the previous evolution. This being the case, as both mass-loss rates and 3DUP efficiency are poorly known, the location of theoretical post-AGB tracks, and thus mass determination, would be highly model dependent and uncertain. These issues call for the need of an analysis of the robustness of existing H-deficient post-AGB tracks and for a way of solving the mentioned mass discrepancies

However, no calculation of the importance of these effects was actually presented neither in WH06 nor in Herwig et al. (2006). The lack of consistent calculations to assess to what a degree the location of the post-AGB tracks depends on the prior AGB evolution has motivated us to undertake the present investigation. In the following sections we will elaborate on these issues. In this sense the present work is complementary to that of MA06 where H-deficient post-AGB tracks were presented but no analysis of its robustness was performed. In Sect. 2 we analyze how evolution previous to the PG 1159 stage affects PG 1159 tracks in light of the suggestion presented by WH06. Then in Sect. 3 we explore to what an extent the constitutive physics of the models at the PG 1159 stage may affect the tracks. In Sect. 4 we compare with other H-deficient tracks available in the literature and also compare the location of tracks coming from LTP and VLTP events. Finally Sect. 5 is devoted to the discussion of the results and making some final remarks.

\section{Influence of previous evolution}

As was mentioned, uncertainties in mass-loss rates are expected to affect the duration of the TP-AGB phase and to lead to remnants with different degrees of degeneracy and mass-luminosity relation. Also the initial-final mass relation is expected to be altered by different mass-loss rates. By altering the intensity of mass loss we can get the same final remnant mass from progenitors of initially very different mass, and consequently very different previous evolutions (e.g., that have or have not undergone a helium core flash at the tip of the RGB). We will address these issues in Sect. 2.1. In Sect. 2.2 we elaborate on the effects of different 3DUP efficiencies on the TP-AGB, which is the other
Table 1. Values of the final masses of the sequences of this work and the masses derived from the comparison with the "standard" ones (MA06). Stellar masses are in $M_{\odot}$. See Sects. 2.1, 2.2, and 2.3 for definition of the sequences.

\begin{tabular}{ccc}
\hline \hline Sequence & Final mass & $\begin{array}{c}\text { Mass derived } \\
\text { from comparison }\end{array}$ \\
\hline NALT & 0.607 & 0.612 \\
LALT & 0.6035 & 0.614 \\
SALT & 0.6033 & 0.598 \\
2.2MSALT & 0.5157 & 0.524 \\
TPA008 & 0.617 & 0.621 \\
TPA004 & 0.633 & 0.637 \\
$3 M_{\odot}$ w/NOV & 0.626 & 0.623 \\
\hline
\end{tabular}

point mentioned in WH06 as a possible cause for shifts in postAGB tracks. The main effect of 3DUP efficiency is to change the initial-final mass relation. Indeed, strong dredge up events on the TP-AGB lead to lower final remnant masses for the same initial mass. In this context we analyze sequences with different 3DUP efficiencies and, to disentangle this effect from the one studied in Sect. 2.1, with the same TP-AGB lifetime. Finally, Sect. 2.3 is intended to clarify the reason of the difference between MA06 and Blöcker (1995a,b) tracks and to study the extreme limiting case for which no overshooting $(\mathrm{OV})$ is allowed to operate at any convective boundary during the whole evolution. In all the sequences presented in this section, mass loss has been arbitrarily set during the departure from the AGB as to get a VLTP and the subsequent PG 1159-like surface composition.

To visualize and quantify the change introduced by the variations in the parameters of each sequence we will refer and compare our sequences with those of MA06 and Córsico et al. (2006) which are assumed as standard in the present work. These sequences were calculated with an overshooting efficiency of $f=0.016$ at all convective boundaries; see Herwig et al. (1997) for a definition of $f$. To quantify the change that a variation in $T_{\text {eff }}$ and $g$ for a sequence of a given mass - caused by different physical assumptions in the calculations - would produce in spectroscopical mass determinations, we estimate a mass value for the sequence from its location relative to MA06 sequences this is what is called "mass derived from comparison" in Table 1 - and compare that mass with the actual value of the mass. The difference between both values gives the shift expected in spectroscopical masses if tracks with a different physical assumption are used in their derivations.

It is worth noting that most of our article deals with postVLTP sequences. However, within the late helium flash scenario for explaining the origin of PG 1159 stars, these objects could also be the offspring of LTP events. In fact some PG 1159 stars are known to be ${ }^{14} \mathrm{~N}$-deficient, a fact usually asociated with postLTP objects. In these cases some $\mathrm{H}$ will be present but hidden below the detection limit. If systematic differences exist between post-LTP and post-VLTP tracks, then this will introduce a systematic effect in spectroscopic mass determiations. Although from Fig. 1 of Herwig one is tempted to conclude that this is not the case, it is worth noting that the presence of $\mathrm{H}$ should be more important in the low mass region as these stars display thicker H-envelopes. We will discuss this issue in Sect. 4.3, where detailed comparisons between post-LTP and post-VLTP tracks will be made for a wide range of masses and various surface $\mathrm{H}$-abundances. 


\subsection{Effect of different TP-AGB lifetime or mass-loss rate}

As stated in WH06, for a similar core mass, a remnant that spend more time on the TP-AGB will finish with a more compact and degenerate core. Then, different mass-loss prescriptions can lead to remnants with the same core mass but different radius and, by virtue of shell homology relations - that "predict" $L_{\text {shell }} \sim M_{\text {core }}^{2} R_{\text {core }}^{-1}$, Herwig et al. (1998) - different luminosities. This is supported by the work of Herwig et al. (1998) that shows that, because of the continuous shrinking of the $\mathrm{H}$-free core (HFC), the luminosity at the TP-AGB keeps increasing, despite the end of the effective core mass growth as consequence of strong dredge up events. In addition, Blöcker (1995b) has already shown that a more compact remnant is more luminous than a less compact one of similar mass. To analyze the possible shift in the H-deficient post-AGB tracks resulting from uncertainties in TP-AGB mass loss - and hence in different TP-AGB lifetimes - we have calculated the full evolution of three sequences with the same prescriptions as in MA06 but changing mass loss at the TP-AGB to get different TP-AGB lifetimes. These sequences are: NALT with a normal mass-loss prescription, SALT with a short TP-AGB lifetime and LALT with a longer TP-AGB. All these sequences come from the same pre TP-AGB evolution of an initially $3-M_{\odot}$ ZAMS star. While NALT underwent 12 thermal pulses, SALT and LALT sequences experienced 6 and 18 pulses, respectively. SALT (LALT) sequence has a TPAGB lifetime a factor 2 shorter (1.5 longer) than NALT. Thus the sequences considered here take into account possible uncertainties in TP-AGB lifetimes up to a factor three. This is more than what is expected from different mass-loss prescriptions (Kitsikis $\&$ Weiss 2007). Due to the high dredge up efficiency during the last thermal pulses the final remnant mass of all these sequences is very similar (see Table 1), thus allowing a direct comparison of the effect of different TP-AGB lifetimes on the location of $\mathrm{H}$-deficient post-AGB tracks of similar mass. We mention that all of these sequences have been followed through an additional post-AGB thermal pulse (the VLTP) where the H-rich envelope is violently burned.

In some agreement with Blöcker (1995b) we find a shift in post-AGB tracks as a consequence of different TP-AGB lifetimes. However the effect is not very important. In fact, comparing SALT and LALT sequences (both with the same final mass) we see that a factor 3 in TP-AGB lifetimes leads to a maximum shift of 0.03 dex in $\log T_{\text {eff }}$. A shorter TP-AGB leads to cooler tracks that would imply $\sim 0.015 M_{\odot}$ larger spectroscopical masses. It is also worth noting that tracks for LALT and NALT sequences are almost identical regardless the difference in TPAGB lifetime of $50 \%$. It seems that, while shortening the TPAGB does change the post-VLTP tracks, prolonging it does not produce a sizeable effect. To understand this, we show in Figs. 1 and 2 the mass-radius relations of our sequence for both the $\mathrm{H}$ and He- free cores - HFC and HeFC, respectively. The evolution of the HFC is in agreement with that presented by Herwig et al. (1998) which shows that the HFC continues to contract even when the core mass growth is stopped by efficient 3DUP events. Because this behaviour is the basis of the argument of WH06 the following should be noted. First the radius of the HFC at the moment of the VLTP does not follow the trend during the TP-AGB. This is a result of the accelerated compression of the intershell caused by the decline of the H-burning shell when the star approaches the white dwarf cooling track. Second, and more importantly, the post-VLTP sequences are powered by the Heburning shell and consequently, if shell homology are to be used in the analyzes, the relevant values should be the HeFC mass and

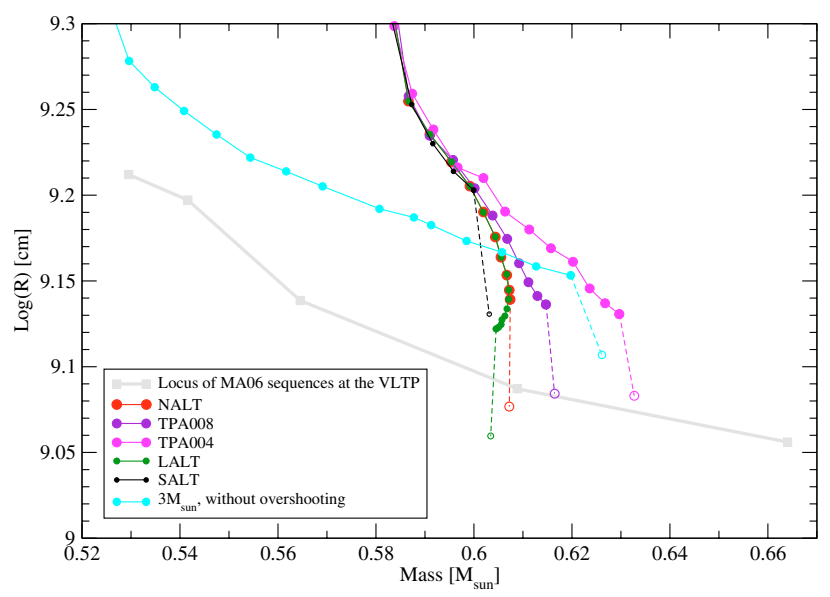

Fig. 1. Evolution of the HFC (mass and radius) during the TP-AGB (solid lines, filled circles) and at the VLTP (dashed lines, empty circles) for selected sequences (values are taken just before each thermal pulse). Also the locus of the standard models at the moment just before the VLTP is shown for comparison. Note that, due to the turn off of the Hburning shell, compression before the VLTP does not follow the trend in the AGB. (Color figure only available in the electronic version.)

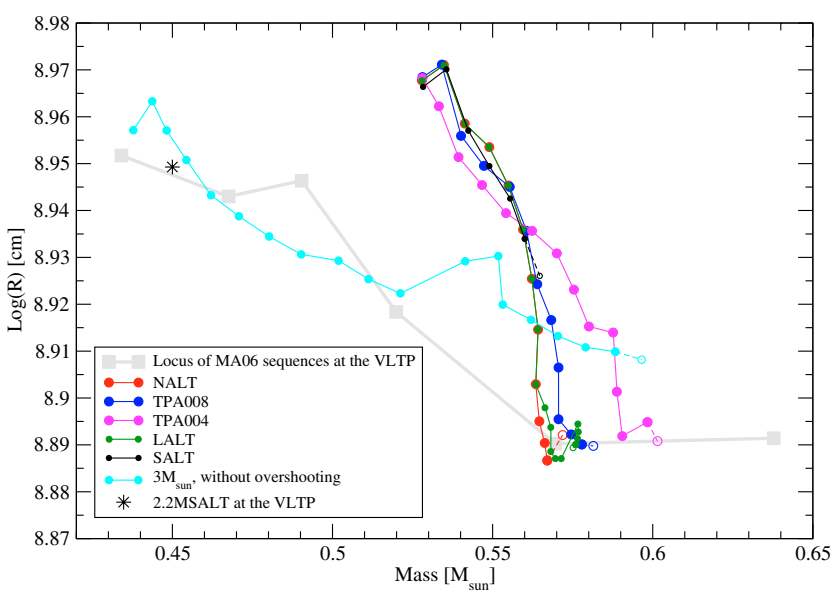

Fig. 2. Same as Fig. 1 but for the HeFC. Note that the location of the $\mathrm{HeFC}$ on this diagram seems to converge, after not many thermal pulses, to a certain locus. (Color figure only available in the electronic version.)

radius. Note that the HeFC (Fig. 2) seems to converge to a certain locus in the core mass-radius diagram faster than the HFC. In fact while in all the sequences the HFC radius gets smaller with each thermal pulse, the HeFC ends its compression after about $\sim 10$ thermal pulses. This helps to understand why there is almost no difference between NALT and LALT sequences. The 6 "extra" thermal pulses of LALT sequence do not introduce any significant change in the mass-radius relation and thus, according to shell homology relations, their post-AGB luminosity should be similar.

As mentioned early, different mass-loss rates can also change the initial-final mass relation of the sequences, leading to final remnants with very different previous evolution but similar final mass. In this connection, we have computed the evolution of an initially $2.2-M_{\odot}$ sequence by assuming an extreme mass-loss rate during the whole AGB (sequence 2.2MSALT). As a result, this sequence underwent only 5 thermal pulses on the AGB as compared with the $15 \mathrm{AGB}$ pulses of the $2.2-M_{\odot}$ sequence in MA06. The final mass of the remnant is of $0.516 M_{\odot}$, much lower than the $0.565 M_{\odot}$ quoted in MA06. The track for this 


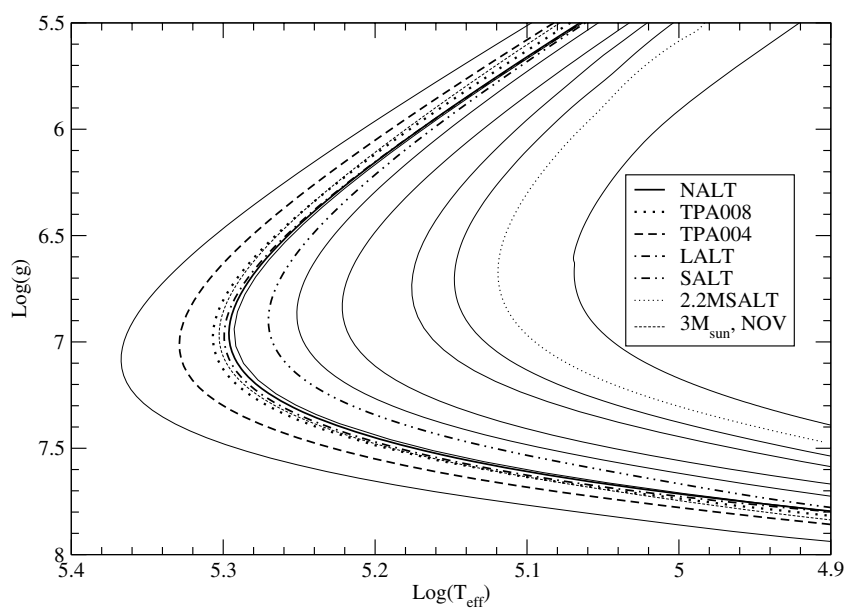

Fig. 3. PG 1159 tracks of this work as compared with those of MA06. Thin solid lines correspond to the standard $(f=0.016$ at all convective borders) tracks of MA06 with stellar masses of (from right to left) $0.512,0.53,0.542,0.565,0.585,0.609,0.664 M_{\odot}$.

sequence in the $\log T_{\text {eff }}-\log g$ plane is shown in Fig. 3 together with the other sequences of this work and those of MA06. Note that the 2.2MSALT track is more luminous and hotter than that of the standard sequence of similar mass (the $0.512 M_{\odot}$ sequence in MA06). Note that in this case, the shift in the $M-g-T_{\text {eff }}$ relation of the remnants would imply a decrease of $\sim 0.01 M_{\odot}$ in spectroscopical masses. This value is unexpectedly low in view of the fact that the two standard sequences in the same region of the $\log T_{\text {eff }}-\log g$ diagram have a very different previous evolution. Indeed, the 0.512 and $0.53 M_{\odot}$ sequences in MA06 have been calculated from an initially $1-M_{\odot}$ progenitor that went through the helium core flash. Also, the $0.512 M_{\odot}$ has a very different intershell and surface composition with only $2 \%$, by mass, of oxygen; see MA06 for a description of this sequence. Again, it is interesting to look at the structure of the HeFC to understand this change. As can be seen in Fig. 2 (black star symbol), although the mass and radius of this model fall almost in the standard locus (the thick grey line in Fig. 2$)$, its HeFC mass $\left(\sim 0.45 M_{\odot}\right)$ is significantly higher than that of the standard $0.512 M_{\odot}$ sequence $\left(\sim 0.43 M_{\odot}\right)$ and thus should be, again from shell homology arguments, more luminous than the standard sequence. Indeed that is what actually happens. Even more, the 2.2MSALT sequence has a HeFC mass that falls almost in the middle of that of the 0.512 and $0.53 M_{\odot}$ MA06 sequences and its track in the $\log T_{\text {eff }}-\log g$ diagram does exactly the same. These considerations seem to support the idea that is the HeFC structure - and not the HFC which is important to understand H-deficient post-AGB tracks.

So, although the findings of this section confirm that different TP-AGB lifetimes may result in changes in the post-AGB tracks, we find that this effect is not enough to account for the mass discrepancy mentioned in the introduction. Indeed, we find that the PG 1159 spectroscopical masses inferred from the MA06 postAGB tracks would be higher by at most $\sim 0.015 M_{\odot}$ (for stars close to the $0.6 M_{\odot}$ tracks) if in their calculations MA06 had considered much shorter TP-AGB lifetimes during the progenitor evolution of their PG 1159 sequences. On the other hand we find impossible to get a similar shift for stars close to the $0.512 M_{\odot}$ track. This is so because the lack of 3DUP in low mass stars.

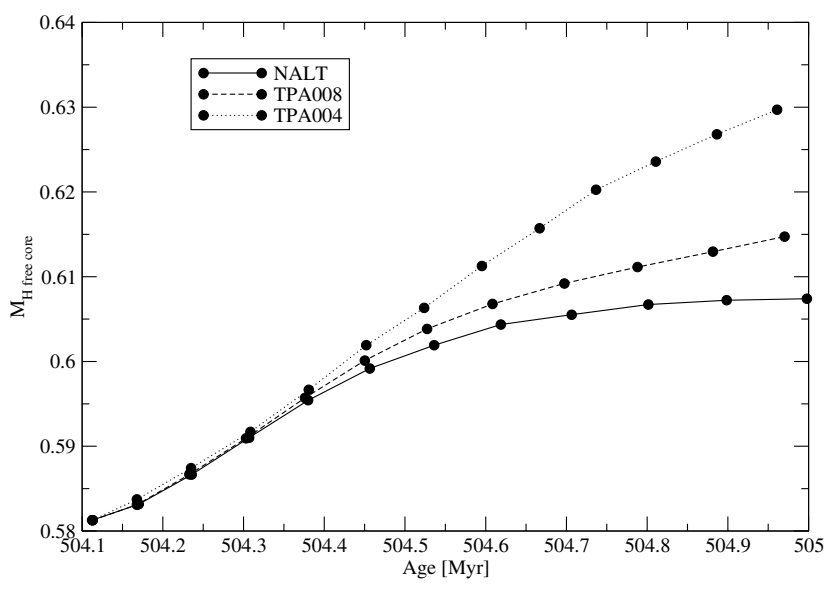

Fig. 4. HFC evolution during the TP-AGB for three sequences with different $f$ values at the PDCZ (masses in $M_{\odot}$ ).

\subsection{Effect of the third dredge-up efficiency}

To explore the role of the 3DUP efficiency during the TP-AGB in the location of post-AGB tracks, we have followed the TPAGB evolution for three different values of the overshooting efficiency $(f)$ at the pulse driven convection zone (PDCZ) that develops during each He-shell flash. As shown in Herwig (2000), higher $f$ values at the bottom of the PDCZ lead to more intense helium shell flashes and more intense third dredge up events, while the value of $f$ at the base of the convective envelope only plays a secondary role in determining the 3DUP efficiency (the reasons for this are extensively discussed and shown in Sects. 4 and 5 of Herwig 2000). We have, thus, calculated three different sequences for a $3-M_{\odot}$ progenitor by adopting values of $f=0.016,0.008,0.004$ at both convective borders of the PDCZ, from now on sequences NALT, TPA008 and TPA004; sequence NALT corresponds to that previously described. At any other convective zone - for example the AGB convective envelope and the core burning regions in the previous evolution - the "standard" value of $f=0.016$ has been used. We stress that the "standard" value $f=0.016$ comes from the fitting of the width of the main sequence (Herwig et al. 1997), and thus is appropriate for the core H-burning zone. But it may be unrealistic for the conditions at the PDCZ (Herwig 2004). All of these sequences have similar TP-AGB lifetimes. This enables us to disentagle the 3DUP effect from the one coming from different TP-AGB lifetimes, which was studied in the previous section. Also, for these three sequences, mass loss during the last interpulse phase has been artificially set in order to obtain a VLTP and consequently a H-deficient post-AGB remnant.

Note from Fig. 4 that different values of $f$ yields different evolution of the HFC. For models with higher 3DUP efficiencies the "effective" growth of the HFC is stopped. This is because the increase in the HFC induced by the H-burning shell is compensated for by a decrease during the 3DUP events. Not only the HFC mass is altered but also, as expected, the surface and intershell abundances - in particular the $\mathrm{O}$ intershell abundance; see Herwig (2000). As a result of the different adopted 3DUP efficiencies, the final remnant masses are different, being $0.607,0.617$ and $0.633 M_{\odot}$ for NALT, TPA008 and TPA004 respectively. It is worth noting that TPA004 hardly undergoes any 3DUP events. So this sequence should be representative of the case in which no overshooting is considered at the PDCZ.

Our results suggest that different 3DUP efficiencies do not seem to lead to an important shift in the location of the 
theoretical post-AGB models in the $M-g-T_{\text {eff }}$ space. Indeed, sequences TPA008 and TPA004 are located in the zone of the log $T_{\text {eff }}-\log g$ diagram corresponding to remnants of similar mass of the standard sequences; see Fig. 3. A quantitative measure of the possible shift in the tracks relative to the standard MA06 ones is given Table 1. Note that there is a small shift of $0.005 M_{\odot}$ in the derived mass for the NALT sequence as compared to the actual one - we remind that NALT sequence has the same overshooting prescription than that assumed in MA06. This is probably due to a combined effect of a different number of thermal pulses and slightly different envelope composition - which leads to different intershell opacities, see Sect. 3. Because the three sequences have similar TP-AGB lifetimes, this small shift should be taken as the level of uncertainties in these comparisons. Keeping this in mind, the masses derived for TPA008 and TPA004 are practically similar to the actual masses of these sequences. This shows that, at least around $\sim 0.6 M_{\odot}$, the theoretical $M-g-T_{\text {eff }}$ relation of the MA06 H-deficient post-VLTP sequences does not seem to depend on the intensity of 3DUP events. This can be understood from Fig. 2. Note that HeFC mass-radius values of sequences TPA008 and TPA004 at the moment of the VLTP lie on the same locus than the standard MA06 sequences of similar masses. Thus, according to shell homology relations, the He-shell luminosity-mass relation for these sequences should be similar to the MA06 ones - which do experience efficient 3DUP events. Finally, we mention that the central values of density and temperature $\left(T_{\mathrm{c}}, \rho_{\mathrm{c}}\right)$ show that the HeFC readjusts its structure to the new mass after each thermal pulse. At the end of the TPAGB the $T_{\mathrm{c}}, \rho_{\mathrm{c}}$ values of TPA004 are within those of NALT - of final HeFC mass $0.572 M_{\odot}$ - and those of the $3.5 M_{\odot}$ sequence of MA06 - of final HeFC mass $0.638 M_{\odot}-$, a fact which is consistent with the final HeFC mass of $0.601 M_{\odot}$ that characterizes sequence TPA004.

\subsection{Evolution previous to the $A G B$}

We explore now the effect of overshooting efficiency during both the early AGB and the core He-burning phase on the location of the post-AGB tracks. This bears also some relevance on the fact that, as inferred from the two previous sections, neither the TP-AGB lifetime nor the 3DUP efficiency are the reasons for the fact that the MA06 tracks are markedly hotter than the older H-rich tracks (Blöcker 1995b). To assess these issues, we have calculated the evolution of an initially $3-M_{\odot}$ progenitor but without overshooting mixing at any convective border of the star during its whole evolution. After 19 thermal pulses, a H-deficient post-VLTP sequence of $0.626 M_{\odot}$ is obtained - early AGB and TP-AGB lifetimes are $\sim 4.8 \times 10^{7} \mathrm{yr}$ and $\sim 2.1 \times 10^{6} \mathrm{yr}$, respectively. This is similar to one of the sequences of Blöcker (1995b) that consisted of an initially $3-M_{\odot}$ model that after 20 thermal pulses ends its post-AGB evolution as a $0.625 M_{\odot}$ remnant with early AGB and TP-AGB lifetimes of $\sim 7 . \times 10^{7} \mathrm{yr}$ and $\sim 1.9 \times 10^{6} \mathrm{yr}$, respectively - and will allow us for comparison. The main evolutionary difference between both sequences is the occurrence of a VLTP in the post-AGB evolution of our sequence.

The resulting H-deficient post-VLTP track is very similar to the MA06 one and thus much hotter than the old, H-rich, Blöcker's $0.626-M_{\odot}$ track. In fact if we estimate its mean mass from comparison with the standard MA06 sequences we get almost the actual mass (see Table 1). The mayor difference is that the model is slightly cooler at the knee - a shift that would affect spectroscopical masses less than $\sim 0.01 M_{\odot}$. From Fig. 2 we can see that the evolution of the HeFC mass-radius relation is different from that of the standard sequences. But even in this case the difference in the radius of the He-free core at the moment of the VLTP amounts to only a $4 \%$ as compared with the standard sequences of similar mass. Consequently, it should not be surprising that the tracks are similar.

This shows that the $M-g-T_{\text {eff }}$ relation for the post-AGB tracks is not significantly affected by the previous evolution. Thus, differences in the previous evolution do not seem to provide a possible solution to discrepancy between asteroseismological and spectroscopical masses nor an explanation to the difference with older tracks.

\section{The role of microphysics and composition in the location of post-VLTP tracks}

We explore now the importance of microphysics and chemical compositions. Specifically we assess the effects of changing the equation of state $(\mathrm{EoS})$, chemical composition of the C-O core and opacities - both radiative and conductive. Here, we do not calculate new evolutionary sequences from the ZAMS to the PG 1159 stage; instead we consider some post-VLTP sequences of MA06 and alter their microphysics before entering the PG 1159 stage. We have checked that the models are already relaxed to the new physics well before reaching the knee in the HR and $\log T_{\text {eff }}-\log g$ diagrams. We check this by first doing the changes at different times in the post-VLTP evolution. We find that the tracks do not depend on the exact moment the changes are done, thus suggesting that the structure has already relaxed to the new situation. Additionaly, we estimate the thermal relaxation time of the envelope as $\tau \sim \int_{M_{\text {e.b. }}}^{M_{\star}} c_{v} T \mathrm{~d} m / L_{\star}-$ where $M_{\text {e.b. }}$. stands for the mass coordinate at the bottom of the envelope. We concentrate on the 0.53 and $0.584 M_{\odot}$ remnants of MA06. For these sequences we find that $\tau$ is about one order of magnitude lower than the time it takes the remnants to evolve from log $T_{\text {eff }} \sim 4.6$ to the knee in the HR diagram. In fact, $\tau$ is about 2500 and $1600 \mathrm{yr}$ for 0.53 and $0.584 M_{\odot}$ remnants respectively, as compared with the $\sim 23000 \mathrm{yr}$ and $\sim 12000 \mathrm{yr}$ it takes the remnant to evolve to the knee. This guarantees that the envelope is thermally relaxed at that point.

\subsection{Equation of state and chemical composition of the $\mathrm{C}$-O core}

To analyze the importance of the C-O core composition and EoS we considered the $0.53 M_{\odot}$ post-VLTP sequence from MA06. With regard to the EoS we compare tracks resulting from the use of the standard EoS of LPCODE - see Althaus et al. (2005) for references - with those coming from an updated version of Magni \& Mazzitelli (1979) EoS. The latter is a more detailed equation of state which takes into account non-ideal corrections such as the pressure effects on ionization and includes Coulomb interactions also in the non-degenerate regime - our standard EoS only includes Coulomb corrections in the degenerate regime. To analyze the role of the core composition we reset the abundances below $\ln \left(1-m(r) / M_{\star}\right)=-2.4-$ thus not altering the composition at the He-burning shell - to two extreme values: $92 \%$ of $\mathrm{C}$ and $92 \%$ of $\mathrm{O}$ by mass. This is not consistent with previous evolution but allows us to estimate its importance for post-VLTP tracks.

Both changes in the EoS and the composition of the CO core do not yield significant changes in the post-VLTP tracks. In fact in none of these cases do we find the shift in $\log T_{\text {eff }}$ to exceed 0.01 dex, being generally much smaller. Consequently, neither 
the $\mathrm{C}-\mathrm{O}$ core composition nor the EoS assumed in the computation of post-VLTP sequences play a role in the derivation of spectroscopical masses, and we can discard these two factors as possible reasons for a shift in post-VLTP tracks.

\subsection{Opacities}

Because the outer structure of PG 1159 stars is completely ruled by radiative transport of energy, changes in the opacities could yield differences in the tracks. This may be particularly interesting as the sequences of MA06 have been calculated for radiative opacities with a solar scaled metallicity and PG 1159 stars are known to present surface abundances rich in s-process elements and iron deficient (Miksa et al. 2002, WH06). In this regard, by how much the transformation of iron into heavier elements may alter the opacities in PG 1159 is not known - for example, in a different context, Jeffery \& Saio (2006) find differences in the pulsation properties of subdwarf stars depending on whether it is iron or nickel that it is enhanced. Also the exact value of the original metallicity of the progenitor star of PG 1159 is not known. We analyze the effect of changing both radiative and conductive opacities with very different results in each case. Full calculations of the VLTP and post-VLTP by means of consistent opacities are out of the scope of this work, however we can try to get an idea of how much the opacities affect the post-AGB tracks by artificially changing the opacities in the post-VLTP evolution by arbitrary factors or by adopting different opacity tables.

As a result of these experiments we find that for conductive opacities even a change of 3 orders of magnitude do not produce significant changes in the post-VLTP tracks. Quite on the contrary, the tracks are more sensitive to radiative opacity changes. In fact we find that - for both the 0.53 and the $0.584 M_{\odot}$ sequences - increasing the opacities by a factor 1.5 produces a reduction of $\sim 0.04 \mathrm{dex}$ in $T_{\text {eff }}$ and $\sim 0.2 \mathrm{dex}$ in $\log L / L_{\odot}$. Similarly a reduction in the opacity by a factor 0.5 leads to increases of $\sim 0.075 \mathrm{dex}$ and $\sim 0.3 \mathrm{dex}$ in temperature and luminosity, respectively. These are important changes and would clearly affect spectroscopic mass determinations ${ }^{1}$. The shift in the location of post-VLTP tracks due to changes in the opacities is displayed in Table 2, where we show the change in $\log T_{\text {eff }}$ for different values of $g$ and for two different remnant masses $\left(0.53\right.$ and $\left.0.607 M_{\odot}\right)$. Also the induced shift in the mass derived from comparison with the $g T_{\text {eff }}$ values of MA06 tracks is shown. Two things deserves comments. The effect of different radiative opacities is much larger for higher remnant masses and at larger luminosities (i.e. lower gravities). Indeed, note that for the $0.53 M_{\odot}$ remnant an increase in the opacity of $50 \%$ would not produce a shift of more than $0.01 M_{\odot}$ in spectroscopical mass determinations, and for the $0.607 M_{\odot}$ remnant the increase in the spectroscopical mass becomes very important, reaching up to $0.07 M_{\odot}$ at high luminosities. Note also that the shift in $\log T_{\text {eff }}$ is almost the same for the same change in $\kappa$ regardless of the mass.

Due to the importance of this effect we consider interesting to analyze if the effect is due to the value of the opacity at some speciffic region of the star - e.g. the He-burning shell. We proceed then to make localised changes in the opacity and found, against expectations, that it is not the value of the opacity (per unit mass $\kappa$ ) at some particular region that is relevant but the total opacity of the envelope $\left(\int_{\text {envelope }} \kappa \mathrm{d} m\right)$. By looking at the

\footnotetext{
${ }^{1}$ It is worth noting that we do not expect important changes in asteroseismological inferences due to changes in opacities. This is so because asteroseismological determinations are usually based on adiabatic period studies, which are barely affected by changes in the opacities.
}

models, we find that altering the radiative opacity produces almost no change in the structure of the envelope. Then, as $\mathrm{d} T / \mathrm{d} m$ is not altered by changes in $\kappa$, varying $\kappa$ leads to an opposite and proportional change in the luminosity $l(m)$ of the star via the relation

$\frac{\mathrm{d} T}{\mathrm{~d} m}=\frac{-3}{64 \pi^{2} a c} \times \frac{\kappa l}{r^{4} T^{3}}$.

A clue of why only $l$ reacts to a change in $\kappa$ can be obtained from the following simple analytical argument. If we assume that the envelopes of these objects are nearly homological to each other, then we have that under homology changes (with $x=\delta r / r$ ) the change in the pressure and density of a shell is (see Kippenhahn $\&$ Weigert 1990, for a deduction)

$\frac{\delta P}{P}=-4 x, \frac{\delta \rho}{\rho}=-3 x$.

Then if we assume an ideal gas equation of state for the envelope - which is quite correct - we have the additional relation

$\frac{\delta T}{T}=-\frac{\delta \rho}{\rho}+\frac{\delta P}{P}=-x$

Using the equation of the temperature profile (Eq. (1)) by imposing an arbitrary change in the opacity $\delta \kappa / \kappa$ and using

$\delta\left(\frac{\mathrm{d} T}{\mathrm{~d} m}\right)=\frac{\mathrm{d} T}{\mathrm{~d} m} \times \frac{\delta T}{T}$

we get

$\frac{\delta T}{T}=\frac{\delta \kappa}{\kappa}+\frac{\delta l}{l}-4 \frac{\delta r}{r}-3 \frac{\delta T}{T}$

and by using Eqs. (2) and (3) we finally find

$\frac{\delta l}{l}=-\frac{\delta \kappa}{\kappa}$,

which is quite similar to what it is observed in the numerical models - during the horizontal part of the tracks. Let us note that this change in the luminosity does at first order balance (in Eq. (1)) the change in opacity, leaving only second order effects on the factor $\kappa l$ to be balanced by the other factors in Eq. (1):

$\kappa^{\prime} l^{\prime}=\kappa l\left(1+\frac{\delta \kappa}{\kappa}\right)\left(1+\frac{\delta l}{l}\right)=\kappa l\left(1-\left(\frac{\delta \kappa}{\kappa}\right)^{2}\right)$.

Also, note that due to the high powers of $r$ and $T$ in Eq. (1), small changes in these quantities should be enough to balance the remaining second order effects. In fact, when looking at the numerical models all $r(m), P(m), T(m)$ remain almost unchanged by the change in the opacity, being $l(m)$ the only structure variable that undergoes an important variation. The change in $l(m)$ seems to be associated with a change in the energy liberated by the helium burning shell - change which can be attained with almost no change in $T(m)$ due to the extremely high sensitivity of triple alpha reaction rates with temperature - and by a change in the $\mathrm{d} S / \mathrm{d} t$ term of the energy equation - the lower the opacity, the faster the evolution and contraction of the envelope. Sumarizing we can say that altering the radiative opacity of the envelope leads to a similar change in the $l(m)$ profile of the star which balances (at first order) the effects of the opacity change in Eq. (1). It seems that as consequence of this balance only minor changes appear in the other structure variables which remain almost unchanged - this probably reflects the fact that the run of these variables in the envelope of the star is forced by the 
Table 2. Shifts in effective temperature ( $\left.\delta \log T_{\text {eff }}\right)$ induced by changes in $\kappa$ for different values of $g$. The value between brackets is the predicted induced shift in spectroscopical masses (in $M_{\odot}$ ).

\begin{tabular}{cccccc}
\hline \hline Sequence & $g=5.5$ & $g=6$ & $g=6.5$ & $g=7$ & $g=7.5$ \\
\hline $0.53 M_{\odot}$ & 0.0782 & 0.0764 & 0.0754 & 0.0735 & 0.0563 \\
$(\kappa \times 0.5)$ & $(-0.1072)$ & $(-0.0687)$ & $(-0.045)$ & $(-0.0292)$ & $(-0.0188)$ \\
$0.53 M_{\odot}$ & -0.0462 & -0.045 & -0.044 & -0.0432 & -0.0365 \\
$(\kappa \times 1.5)$ & $(0.0096)$ & $(0.0106)$ & $(0.0099)$ & $(0.0086)$ & $(0.0066)$ \\
$0.607 M_{\odot}$ & 0.0788 & 0.0732 & 0.0724 & 0.0724 & 0.0757 \\
$(\kappa \times 0.5)$ & $(-0.1748)$ & $(-0.126)$ & $(-0.0888)$ & $(-0.0545)$ & $(-0.031)$ \\
$0.607 M_{\odot}$ & -0.0455 & -0.0442 & -0.0425 & -0.0418 & -0.0422 \\
$(\kappa \times 1.5)$ & $(0.0684)$ & $(0.0484)$ & $(0.0366)$ & $(0.0243)$ & $(0.0158)$ \\
\hline
\end{tabular}

radius and mass of the He-free core where most of the gravitational field is generated. Although this does not intend to be a complete explanation, something which is impossible nowadays due to the lack of an accepted explanation for the behaviour of structures with burning shells ${ }^{2}$, we think that it sheds some light on what is happening on these models. Finally let us mention that as $r(m)$ is not changed, then the radius of the star is almost the same, independently of the value of the opacity. Then, due to Steffan-Boltzmann's law we have that the change in the opacity produces a variation in the effective temperature of

$\frac{\delta T_{\mathrm{eff}}}{T_{\mathrm{eff}}}=\frac{1}{4} \frac{\delta L_{\star}}{L_{\star}}-\frac{1}{2} \frac{\delta R_{\star}}{R_{\star}} \sim \frac{1}{4} \frac{\delta L_{\star}}{L_{\star}}$.

In fact for the $0.584 M_{\odot}$ models with normal and enhanced (for a factor $\left.1.5, \frac{\delta K}{K} \sim 0.5\right)$ opacity we have that, in the knee, $\frac{\delta L}{L} \sim$ 0.34 and $\frac{\delta T_{\text {eff }}}{T_{\text {eff }}} \sim 0.09$ and thus $\frac{\delta T_{\text {eff }}}{T_{\text {eff }}} \sim \frac{1}{4} \frac{\delta L_{\star}}{L_{\star}}$. Note however that $\frac{\delta L}{L} \sim 0.34$ is far from the value 0.5 in $\frac{\delta \kappa}{K}\left(\frac{\delta L}{L}\right.$ is close to 0.5 in the horizontal part of the track, i.e. $\log T_{\text {eff }}<4.8$ ).

\subsection{Effect of different compositions on the opacities}

As models seem to be sensitive to the value of the radiative opacity in the envelope, we have analyzed how much opacities can change due to different adopted compositions.

Firstly, we have assessed possible changes in the tracks due to changes in the total amount of metals in the models - with the exception of $\mathrm{C}$ and $\mathrm{O}$ which are always kept consistent with the envelope abundance. We did this by using OPAL C-O-enhanced tables for $Z=0.01$ and $Z=0.03$ - all of our previous sequences correspond to $Z=0.02$. The change in metallicity was done well before evolution reached the PG 1159 stage. We find that the resulting shifts in the tracks are barely noticeable. In fact, at the knee in the HR diagram the $Z=0.01$ and $Z=0.03$ tracks differ in effective temperature by only $\sim 0.006$ dex.

Secondly, we have explored the use of opacities fully consistent with the abundances of the models. This is not a minor issue as $\mathrm{Ne}$ and $\mathrm{N}$ can be much larger than their solar scaled values - also $\mathrm{Mg}$ can reach values of $2 \%$; see Werner et al. (2004). Specifically, we have used the tool at OP project website (Badnell et al. 2005) which allows to calculate opacities for arbitrary compositions. In this case we have not made any track calculation but instead we have just compared the opacities for a given model (i.e. for a given $T$ and $\rho$ profile). We compare first OP and OPAL opacities for the same imput composition. The result is shown in Fig. 5 Note that, for $\log T>7$, OP opacities tend to be about 5 to $7 \%$ larger. This would probably introduce

\footnotetext{
${ }^{2}$ In fact the problem is in some way related with the long standing problem of why stars become red giants; see Faulkner (2005) for a recent discussion, and review, of this issue.
}

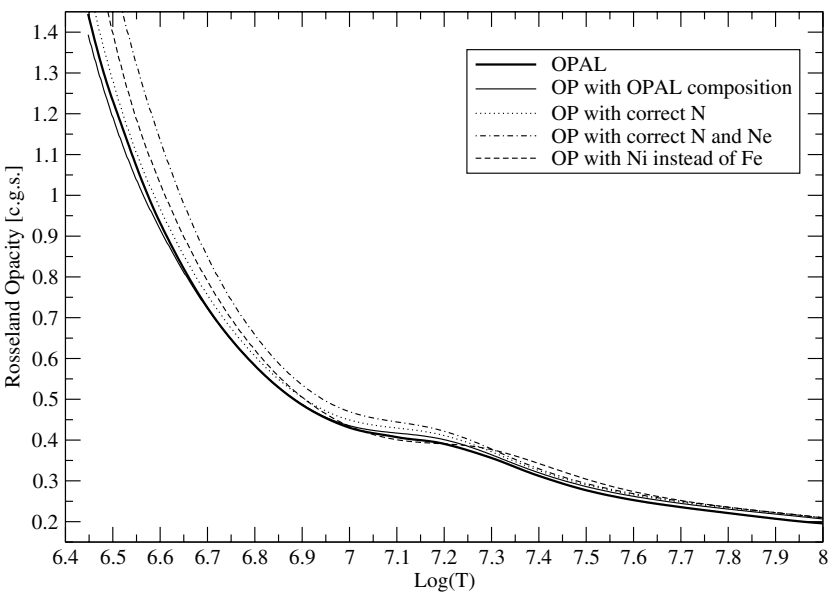

Fig. 5. Value of the opacity for different adopted compositions.

a small change of about 0.01 dex in the tracks. The inclusion of $\mathrm{N}$ and $\mathrm{Ne}$ - with abundances consistent with those displayed by the stellar models - markedly increases the opacity values below $\log T \sim 7.3$, but almost no changes are present at higher temperatures where most of the mass of the envelope is stored, see Fig. 5. As PG 1159 stars are supposed to be iron deficient due to s-process (WH06), we have analyzed the extreme case for which all the iron was changed into Ni. In this case, the opacity bump is located at larger $T$ values, thus increasing opacity between $\log T=7.3$ and 7.6. Although this change in the opacity is not enough to reconcile the discrepancy between spectroscopical and asteroseimic masses of PG 1159 stars, it is important to note that modifying the heavy metal distribution does introduce a change in the opacities at high temperatures. Indeed, as opacities increase with the atomic number of the elements - due to the increase in the possible atomic transitions, Roger \& Iglesias (1994) - it remains to be seen to what a degree an important increase in the content of very heavy metals due to s-process (both in the AGB and at the VLTP) can increase the opacity at the bottom of the envelope. Note that because of the higher ionization potentials, those elements are expected to affect opacities at much higher temperatures than do Fe or Ni.

\section{Other issues}

\subsection{External consistency}

We have compared our tracks with the few models of modern H-deficient post-AGB sequences available in the literature, particularly the $0.604 M_{\odot}$ model of Herwig (2005) and the $0.632 M_{\odot}$ model of Lawlor \& Mac Donald (2006).

Note from Fig. 6 that our models show a good agreement with the tracks of both authors. The agreement is remarkable 


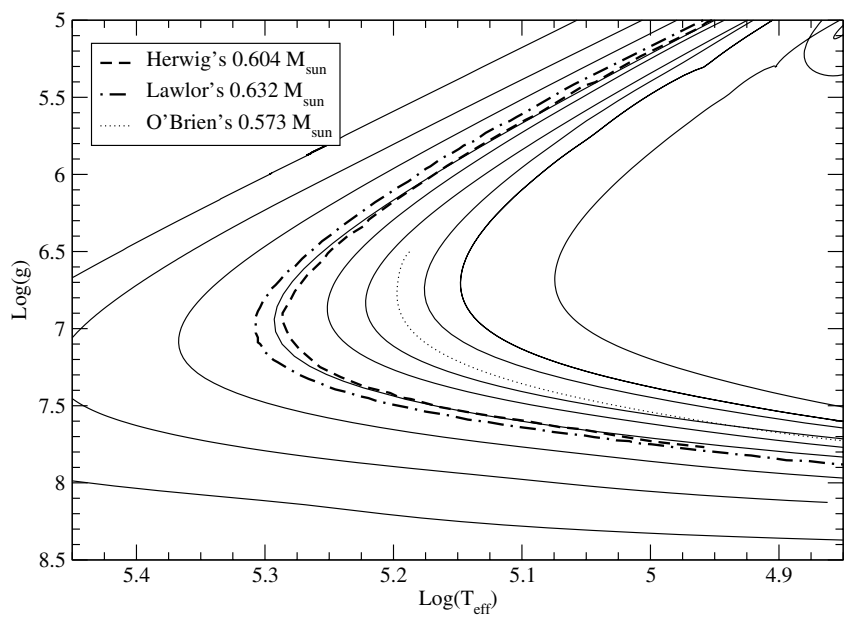

Fig. 6. H-deficient tracks of Herwig (2005) and Lawlor \& Mac Donald (2006) compared with our post-VLTP tracks (with masses $0.87,0.741$, $0.664,0.609,0.585,0.565,0.542,0.530$, and $\left.0.515 M_{\odot}\right)$. Also a nonlate helium flash (but H-deficient) track from O'Brien (2000) is shown for comparison.

despite the very different input physics and evolutionary history of progenitor stars considered by those authors. Indeed, Lawlor \& Mac Donald (2006) models do not include any kind of overshooting prescription and Herwig's model is the result of an initially $2 M_{\odot}$ star model and thus with a distinct previous evolution than our $0.609 M_{\odot}$ sequence which comes from a $3 M_{\odot}$ model. In addition, the EoS are different in all the cases. This supports the findings in Sects. 2 and 3. For a quantitative inference, we estimate masses for those tracks by comparing their relative location with MA06 tracks. We derive masses of about $0.611 M_{\odot}$ and $0.623 M_{\odot}$ for Herwig and Lawlor \& Mac Donald sequences respectively - note that the resulting Herwig's track becomes bluer than ours, leading to slightly lower spectroscopical masses. In both cases the induced shift in spectroscopical masses would be lower than $0.01 M_{\odot}$, thus reinforcing the robustness of the MA06 post-AGB tracks.

\subsection{Comparison with $\mathrm{H}$-burning tracks}

As mentioned early in this work H-burners old tracks are cooler than MA06 tracks, thus leading to a much better agreement with asteroseismology (WH06). As we discussed in Sect. 2, the difference in the location between old and new tracks cannot be tracked back to a distinct previous evolution, with the exception of the VLTP event. It is worth noting that old models (Blöcker 1995a,b) are based on the Cox \& Stewart (1970) opacities, in contrast to new models that use OPAL and molecular opacities. In this connection we feel important to recall that already Dreizler \& Heber (1998) noted a shift of $0.03 M_{\odot}$ between old Wood \& Faulkner (1986) and O'Brien \& Kawaler (as they appear in Dreizler \& Heber 1998, here shown in Fig. 6) heliumburning tracks, where the latter make use of modern OPAL opacities. Appart from possible changes that could arise from the different opacities used in the calculations, the difference in the tracks can be expected from the very fact that the Blöcker's tracks are H-burners while VLTP are helium burners. In fact, we note from our sequences that there is a noticeable difference in H-burning-post-AGB and post-VLTP tracks for low remnant masses $\left(\leqslant 0.53 M_{\odot}\right)$. This can be seen in Fig. 7, where we show our post-AGB H-burning tracks (with H-rich surface compositions) of 0.517 and $0.53 M_{\odot}$ compared with the post-VLTP

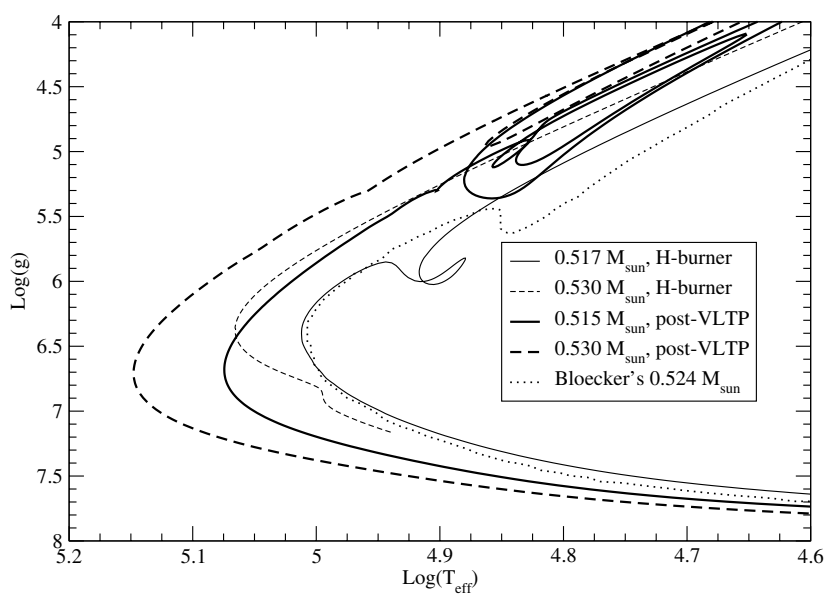

Fig. 7. Comparison between H-rich, H-burning tracks and H-deficient, He-burning tracks of low mass. It is clear from the figure that H-burners have lower $T_{\text {eff }}$ and are much similar to Blöcker's tracks. Using H-rich tracks to estimate PG 1159 masses can lead to important overestimations in the low mass range.

tracks of similar mass. Note that post-VLTP tracks are certainly bluer than their H-rich counterparts and that they are more compact in the high gravity region of the diagram $(\log g>6.5)$, as found by MA06. Also note from the same figure that our 0.517 $M_{\odot}$ track is very similar to Blöcker's $0.524 M_{\odot}$ track, strongly suggesting that the difference with Blöcker (1995b) tracks at (very) low masses is because MA06 tracks are post-VLTP tracks. Using H-rich tracks to determine spectroscopical masses for PG 1159 stars will certainly influence the result, in particular the stellar masses for high-gravity PG 1159 stars would be largely overestimated.

\subsection{Comparison of post-LTP and post-VLTP tracks}

As mentioned early in this work, ${ }^{14} \mathrm{~N}$-deficient PG 1159 objects are probably the descendents of LTP events. Thus, a priori one should be careful about using post-VLTP tracks for all PG 1159 stars. In this context we now turn to analyze the question if there are systematic differences between post-VLTP and postLTP tracks. From Fig. 1 of Herwig 2001 it seems that there is no differece once the star enters the PG 1159 stage. However we will now analyze a wider range of masses. In Fig. 8 PG 1159tracks coming from VLTP and LTP are compared for similar remnant masses. In the upper panel LTP tracks with different $\mathrm{H}$-abundances are compared with VLTP tracks of similar mass. The $\sim 0.515 M_{\odot}$ tracks correspond to the sequence analysed in Althaus et al. (2007). In these sequences two different post-LTP evolutions have been considered. The first in which the final surface $\mathrm{H}$-abundance is normal ${ }^{3}$ and a second in which due to mass loss episodes the whole H-rich envelope was eroded, exposing the He-rich intershell. Due to the absence of the H-burning shell in the second case it lies very close to the postVLTP tracks. The second experiment is also shown in the upper panel of Fig. 8 corresponds to an LTP sequence $\left(0.543 M_{\odot}\right)$ in which the total $\mathrm{H}$ content of the star was artificially diluted to different depths, thus leading to different final surface $\mathrm{H}$-abundances. As can be seen once the star reaches the PG 1159 stage, the lower the surface $\mathrm{H}$ abundance the closer the LTP-tracks gets to the VLTP track of similar mass. Finally, in the lower panel of Fig. 8 post-LTP tracks

\footnotetext{
${ }^{3}$ In this sequence, due to the very low remnant mass, the low intensity of the He-flash does not lead to any 3DUP.
} 


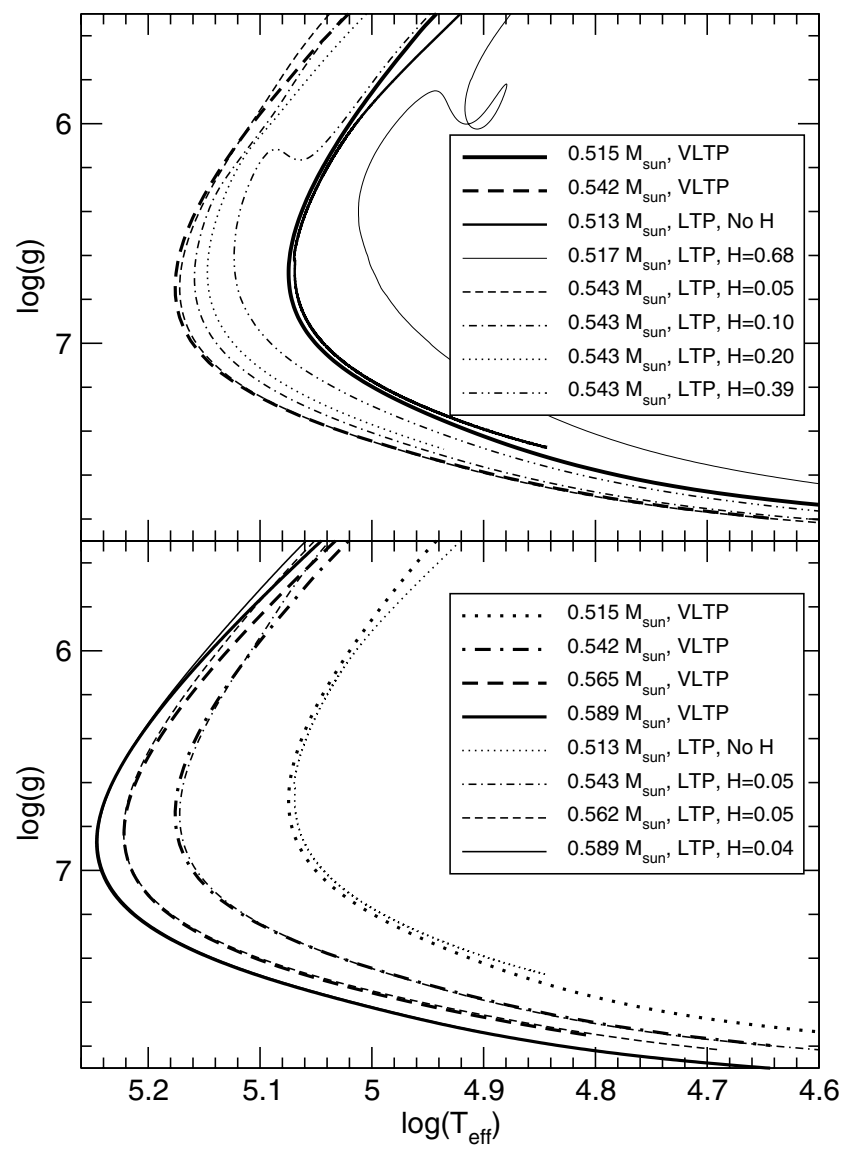

Fig. 8. Comparison between post-LTP and post-VLTP tracks. Upper panel: comparison between VLTP tracks and post-LTP tracks of similar mass but different $\mathrm{H}$ abundances. Lower panel: comparison between post-VLTP tracks with post-LTP tracks that display surface hydrogen abundances, close to the detection limit.

of $\mathrm{H}$ abundances close to the usual detection limit are compared with VLTP tracks of similar mass. From that plot is clear that for surface gravities above $\log g=6$, where almost all PG 1159 stars lie, VLTP tracks and LTP tracks with low $\mathrm{H}$-abundances are similar. Then using post-VLTP tracks for spectroscopic mass determinations of LTP objects with no detectable $\mathrm{H}$ does not seem to introduce any systematic effect on the mass determination. On the contrary using post-VLTP tracks for hybrid PG 1159 stars may produce an important underestimation of the mass.

\section{Discussion and final remarks}

In the present work we have analyzed how uncertainties in the modeling of H-deficient post-VLTP remnants could affect spectroscopic mass determinations of PG 1159 type stars. In Sect. 2, inspired by a suggestion in WH06 we have analyzed the importance of previous evolution. As the calculation of each full sequence is extremely time consuming (both computational and human, as at some stages the models need hand interaction to converge them) we had to restrict ourselves to a limited region of the parameter space. Even in this case some conclusions can be drawn. Third dredge up alone does not seem to change the theoretical $\log T_{\text {eff }}-\log g$ - $M$ locus and consequently its uncertainties can not affect spectroscopic mass determinations. On the other hand differences in mass loss rates (i.e. TP-AGB lifetimes) alter the location of the tracks, but only slightly. In fact our simulations show that even a reduction by a factor of 3 of
TP-AGB lifetimes would not increase spectroscopic mass determinations by more than $\sim 0.015 M_{\odot}$. Going even further we have shown that even extreme mass losses that produce low mass remnants $\left(\sim 0.515 M_{\odot}\right)$ from very different progenitors than those in MA06, does not introduce important shifts in spectroscopic mass determinations, being only $\sim 0.01 M_{\odot}$. In an even more extreme limiting case we have computed a sequence, in which no overshooting was considered in the whole evolution, and found a very similar post-VLTP track than MA06. All these experiments suggest, contrary to the argument in WH06, that previous evolution only plays a secondary (and not very important) role in determining the theoretical $\log T_{\text {eff }}-\log g-M$ locus. In light of these results we have discussed why the argument presented in WH06 does not apply to post-VLTP sequences. We showed that it is the HeFC (if any), and not the HFC, mass and radius that is important for post-VLTP tracks. In particular we find that the HeFC converges faster than the HFC in the mass-radius diagram. However, shell homology relations (as those used to derive the luminosity-mass-radius relation) should not be taken too seriously in these models, as they neglect the importance of the envelope and only relate the luminosity of the burning shells to the properties of matter in the burning shells and to the values of mass and radius of the core, since we find (in Sect. 3.2) an important dependence of the shell luminosity with the whole opacity of the envelope.

We have roughly addressed the robustness of the tracks regarding EoS, C-O core composition, conductive and radiative opacities. We find that only radiative opacity may affect the location of the tracks to some an extent. Specifycally we find that the luminosity of the post-VLTP sequences in the horizontal part of the HR diagram is very sensitive to the envelope opacity. In fact the luminosity of the He-burning shell turns to be sensitive to the total opacity of the envelope. We also present some analytical arguments to explain the shift induced by changes in radiative opacitites. In this connection we explore how important the envelope composition can be for the opacity of the envelope. We find that changes in light metals ( $\mathrm{Ne}$ and $\mathrm{Mg}$ ) can make important changes in the opacities but only at low temperatures $\left(T<5 \times 10^{7} \mathrm{~K}\right)$ where no much mass of the envelope is stored. Although this may be important for pulsational studies of PG 1159 stars, it will certainly not change the $T_{\text {eff }}$ of the sequences. By contrast changing $\mathrm{Fe}$ into $\mathrm{Ni}$ in the opacity calculations we find a more slightly important change. This particularly leaves open the question of how much opacities at the bottom of the envelope can change if important amounts of Fe are transformed into very heavy metals by s-processes. We can conclude that, unless there are important changes in the abundances of very heavy elements due to s-process, an increase in the opacity at high $T$ is not expected to change more than $10 \%$.

All these arguments show that MA06 tracks are robust enough as to be used for spectroscopical mass determinations of PG 1159-type stars (specially at high gravities; $\log g \gtrsim 6$ ). This robustness is reinforced by the good agreement (which corresponds to differences of $\lesssim 0.01 M_{\odot}$ in spectroscopic mass determinations) between those tracks with the other modern post-VLTP tracks available in the literature (Herwig 2005; and Lawlor \& Mac Donald 2006). We have also addressed in Sect. 4.3 if any systematic in the mass determination may be due to the fact of some PG 159 stars being post-LTP objects with $\mathrm{H}$-abundances below the detection limit. We find that the resulting tracks in the PG 1159 region of the $T_{\text {eff }}-\mathrm{g}(\log g>6)$ diagram are very similar to post-VLTP tracks when surface $\mathrm{H}$ abundance is below $\sim 5 \%$ by mass fraction. Thus, we conclude that the post-VLTP tracks of MA06 are solid enough for spectroscopic 
mass determinations of post-LTP objects with H-abundances below the detection limit and, thus, it seems that no systematic should be present due to this effect. On the contrary, we find that using post-VLTP tracks for PG 1159 stars with important $\mathrm{H}$-abundances (the so called hybrid PG 1159 stars) may lead to an important underestimation of the mass. Regarding the difference with Blöcker's H-rich post-AGB tracks we can say that, for low mass remnants $\left(\leqslant 0.53 M_{\odot}\right)$, the differences in the tracks seem to be mainly due to the fact that those tracks are H-burners since our own H-burner sequences are much colder than our post-VLTP ones. Other differences with older tracks may be due to the difference in the opacities adopted for the He, C-rich intershell (note that older works make use of old Cox \& Stewart opacities). These seems to be supported by the good agreement between all $\mathrm{H}$-deficient tracks that include modern OPAL opacities - Herwig 2005, Lawlor \& Mac Donald 2006 and, more roughly $\left(\sim 0.02 M_{\odot}\right)$, even with the non- late helium flash $0.573 M_{\odot}$ sequence of O'Brien 2000.

From the present work we judge that the systematic discrepancy between asteroseismological and spectroscopical mass determination methods should not be attributed to uncertainties in post-AGB tracks. Whether the discrepancy comes from errors in asteroseismological or spectroscopical determinations is not known, however some points are worth emphasising. Although asteroseismology is usually accepted as a more accurate method (very low error bars are usually given), its robustness is not so clear. In fact recent works (Córsico \& Althaus 2006; Córsico et al. 2007a; Córsico et al. 2007b) show the results of asteroseismology to be method dependent. In this context it is worth emphasising that the asteroseismic mass of PG 1159-035 is reduced to $\sim 0.56 M_{\odot}-$ only $\sim 0.02 M_{\odot}$ higher than its spectroscopical mass - when detailed evolutionary models and averanged period spacing (instead of the usually adopted asymptotic period spacing) are used in the analysis, see Córsico et al. (2006). Interestingly enough, during the referee stage of this article a new study of PG 0122+200 (Córsico et al. 2007b) which is based on our evolutionary models and a detailed period by period fitting procedure, reduces the mass discrepancy (with MA06 value) in this star to less than a $4 \%$. This clearly shows the existence of serious systematics in standard (i.e. based on asymptototic period spacing) asteroseismological determinations. In this context is worth noting that a mean PG 1159 mass of $0.573 M_{\odot}$ like the one deduced from MA06 tracks, even if sensitively lower $\left(0.044 M_{\odot}\right)$ than previously thought, is in good agreement with that of their probable descendants, the DB white dwarfs $\left(0.585 M_{\odot}\right.$, Beauchamp et al. 1996) ${ }^{4}$. Then our results not only call for a revision of PG 1159 model atmospheres but, specially, for a revision of systematics in usually adopted asteroseismological mass determination methods.
Our full set of evolutionary tracks for post-VLTP objects is available at our web site at http://www.fcaglp.unlp. edu.ar/evolgroup/

Acknowledgements. M3B wants to thank Achim Weiss, Agis Kitsikis and Alejandro Córsico for useful and instructive discussions and the Max Planck Institut für Astrophysik in Garching and the European Assossiation for Research in Astronomy for and EARA-EST fellowship during which part of this work was done. This research was partially supported by the PIP 6521 grant from CONICET.

\section{References}

Althaus, L. G., Serenelli, A. M., Panei, J. A., et al. 2005, A\&A, 435, 631 Althaus, L. G., Córsico, A. H., \& Miller Bertolami, M. M. 2007, A\&A, 467, 1175

Badnell, N. R., Bautista, M. A., Butler, K., et al. 2005, MNRAS, 360, 458 Beauchamp, A., Wesemael, F., Bergeron, P., Liebert, J., \& Saffer, R. A. 1996, ASP Conf. Ser., 96, 295

Blöcker, T. 1995a, A\&A, 297,727

Blöcker, T. 1995b, A\&A, 299,755

Córsico, A. H. \& Althaus, L. G. 2006, A\&A, 454, 863

Córsico, A. H., Althaus, L. G., \& Miller Bertolami, M. M. 2006, A\&A, 458, 259 Córsico, A. H., Althaus, L. G., Miller Bertolami, M. M., \& Werner, K. 2007a, A\&A, 461, 1095

Córsico, A. H., Miller Bertolami, M. M., Althaus, L. G., Werner, K., \& Vauclair, G. $2007 \mathrm{~b}, \mathrm{~A} \& \mathrm{~A}$, to be submitted

Cox, A. N., \& Stewart, J. N. 1970, ApJS, 19, 243

De Marco, O. 2002, ApSS, 279, 157

Dreizler, S., \& Heber, U. 1998, A\&A, 334, 618

Eisenstein, D. J., Liebert, J., Harris, H., et al. 2006, ApJS, 167, 40

Faulkner, J. 2005, The scientific legacy of Fred Hoyle, ed. D. Gough, 149

Herwig, F. 2000, A\&A, 360, 952

Herwig, F. 2001, ApSS, 275, 15

Herwig, F. 2004, ApJS, 155, 651

Herwig, F. 2005, ARA\&A, 43, 435

Herwig, F., Blöcker, T., Schönberner, D., \& El Eid, M. 1997, A\&A, 324, L81

Herwig, F., Schönberner, D., \& Blöcker, T. 1998, A\&A, 340, L43

Herwig, F., Blöcker, T., Langer, N., \& Driebe, T. 1999, A\&A, 349, L5

Herwig, F., Freytag, B., \& Werner, K. 2006, IAU Symp., 234, 103

Iben, I., Kaler, J. B., Truran, J. W., \& Rensini, A. 1983, ApJ, 264, 605

Jeffery, C. S., \& Saio, H. 2006, MNRAS, 372, L48

Kawaler, S. D., \& Bradley, P. A. 1994, ApJ, 427, 415

Kitsikis, A., \& Weiss, A. 2007, ASP Conf. Ser., in press

Kippenhahn, R., \& Weigert, A. 1990, Stellar Structure and Evolution (Springer-Verlag)

Lawlor, T. M., \& Mac Donald, J. 2006, MNRAS, 371, 263

Magni, G., \& Mazzitelli, I. 1979, 72, 134

Miksa, S., Deetjen, J. L., Dreizler, S., et al. 2002, A\&A, 389, 953

Miller Bertolami, M. M., \& Althaus, L. G. 2006, A\&A, 454, 845 [MA06]

O'Brien, M. S. 2000, ApJ, 532, 1078

Rogers, F. J., \& Iglesias, C. A. 1994, Science, 263, 50

Saio, H., \& Jeffery, C. S. 2002, MNRAS, 333, 121

Werner, K., \& Herwig, F. 2006, PASP, 118, 183 (WH06)

Werner, K., Heber, U., \& Hunger, K. 1991, A\&A, 244, 437

Werner, K., Rauch, T., Barstow, M. A., \& Kruk, J. W. 2004, A\&A, 421, 1169

Wood, P. R., \& Faulkner, D. J. 1986, ApJ, 307, 659
4 This is just a rough comparison as neither all PG 1159 stars are expected to evolve into DB white dwarfs nor all DB white dwarfs are expected to be the result of single stellar evolution; e.g. see Saio \& Jeffery (2002). 